\title{
Challenges of STEM CELl APPlication in RESEARCH AND CLINICAL PRACTICE - AN UPDATE
}

\author{
Maurycy Jankowski ${ }^{1}$, Marie Machatkova ${ }^{2}$, Pavel Ventruba ${ }^{3}$, Elena Kistanova ${ }^{4}$, Alexander Makarevich ${ }^{5}$, \\ Radek Prochazka ${ }^{6}$, Michal Jeseta ${ }^{7,3}$
}

\begin{abstract}
There are multiple possible applications of stem cells in medicine, from cell-based therapies for degenerative and dystrophic conditions, through novel approaches in cancer treatment, to in vitro organ printing. However, there are still several challenges that need to be overcame before stem cells therapies can be successfully introduced worldwide on a large scale. These include sourcing of stem cells, preventing their aberrant progression and ethical concerns regarding their use in animals and humans. Among the multiple stem cell types present in the human organism from the period of embryonic development to adulthood, this review focuses on the three types that gain the most attention in relation to modern research: embryonic stem cells, induced pluripotent stem cells and adult stem cells. There are a number of obstacles that need to be removed before these cells can be widely applied in clinical practice, including the choice of the perfect source of stem cells, full elucidation of the mechanisms of stem cell differentiation and plasticity, and minimization of adverse side effect potential. Nonetheless, the focus of the scientific community on the topic of stem cells remains unhindered, bringing hope that all of the possible concerns will be addressed in the future.
\end{abstract}

Running title: Ethics of stem cell application

Keywords: stem cells, ethical challenges, research, therapy

\footnotetext{
${ }^{1}$ Department of Anatomy, Poznań University of Medical Sciences, Poznań, Poland

${ }^{2}$ Veterinary Research Institute, Brno, Czech Republic

${ }^{3}$ Department of Obstetrics and Gynecology, University Hospital and Masaryk University, Brno, Czech Republic

${ }^{4}$ Institute of Biology and Immunology of Reproduction, Bulgarian Academy of Sciences, Sofia, Bulgaria

${ }^{5}$ National Agricultural and Food Centre, Research Institute of Animal Production Nitra, Nitra, Slovak Republic

${ }^{6}$ Institute of Animal Physiology and Genetics, Czech Academy of Sciences, Liběchov, Czech Republic

${ }^{7}$ Department of Veterinary Sciences, Czech University of Life Sciences in Prague, Prague, Czech Republic

*Correspondence: jeseta@gmail.com

Full list of author information is available at the end of article
} 


\section{Introduction}

In the recent years, a significant scientific effort is devoted to the research regarding stem cells. This is a result of major hopes that such studies may result in crucial improvements to the management and treatment of a range of diseases, as well as the development of new technologies from the field of regenerative and reconstructive medicine. The applications of stem cells in medicine in biotechnology seem almost endless, from cell-based therapies for degenerative and dystrophic conditions, through novel approaches to cancer treatment, to in vitro organ printing [1].

However, there are still several challenges that need to be overcame before stem cells therapies can be successfully introduced worldwide on a large scale. These include sourcing of stem cells, preventing their aberrant progression and ethical concerns regarding their use in animals and humans. The latter is especially important, as societal fears that might arise from the lack of discussion of potential ethical limitations of such approaches might result in slowing down or impeding their introduction on a wider scale [2].

Hence, this review briefly summarizes and discusses the ethical concerns attached to three different groups of stem cells with the most clinical potential and scientific focus: embryonic stem cells (ESCs), induced pluripotent stem cells (IPSCs) and adult stem cells [3].

\section{Embryonic stem cells}

Embryonic stem cells, first described in a laboratory experiment based on mouse embryos in 1981, have since been associated with a significant amount of controversies [1]. Due to the almost highest possible degree of plasticity (pluripotency), they are potentially able to form any type of lineage found in developing and adult human organism when properly induced [4]. Due to this property, the scientific community is vocal about the fact that this type of stem cells shows the biggest promise in treatment of a range of neurological and degenerative diseases, such as stroke, Alzheimer's disease, and Parkinson's disease [5]. However, as the current knowledge of embryonic stem cells deems it unlikely to use animal sourced material in human therapy, the ESCs would need to be sourced from human embryos [6]. This poses a significant barrier to their use for several reasons. Firstly, as the inner cell mass (the source of ESCs) is present and accessible in early embryogenesis, the sourcing of these stem cells would need to be performed on in vitro fertilized embryos. This, in turn, would require oocytes, obtained indirectly from stocks frozen in IVF clinics or directly from consenting donors, or already cryopreserved embryos. Such an approach would certainly be associated with a significant societal pushback, due to personal, religious and ethical reasons [2]. Furthermore, as the ESCs would most likely be sourced from an unrelated donor, there are some significant concerns regarding potential immunogenicity of the cells used during therapy. However, this obstacle could potentially be overcome through creation of cell banks containing a range of human HLA haplotypes [7]. There are also several concerns regarding the pluripotent characteristics of ESCs, which will be discussed in detail in the next section regarding IPSCs, as they are common for both of these stem cell types. Summarizing, while there are some applications in which ESCs could potentially be used, the significant ethical concerns regarding their sourcing, as well as range of potentially downsides related to their pluripotency makes them highly unlikely to be applied in clinical practice.

\section{Induced pluripotent stem cells}

induced pluripotent stem cells (IPSCs) pose significant advantages over ESCs, mostly related to the ethical aspects of embryo culture and manipulation [8]. They are a pluripotent stem cell type which can be generated in in vitro conditions using adult somatic cells. First described in 2006 by Yamanaka et al., they were created from mouse fibroblasts using a combination of 24 factors playing major roles in the plasticity of ESCs. Since then, this knowledge has been translated into human studies, resulting in an accessible source of cells of extraordinary plasticity, able to proliferate indefinitely in laboratory conditions (Fig. 1). [9]. This brought the attention of the international scientific community towards IPSC application in a broad range of regenerative and reconstructive medicine applications. It is also worth noting that cells obtained in such way could be a source of therapeutic lineages used for treating the donor, eliminating further concerns regarding their potential immunogenicity [10]. However, there are several potential downsides that need to be addressed before such cells could be used in a clinical setting. Firstly, while the already generated IPSCs can theoretically proliferate indefinitely, the efficiency of their conversion from somatic cells is still extremely low. This is not a significant obstacle in research, but could pose a problem in treatment, causing the need for collection of a large amounts of source material from the donor [11]. Furthermore, as the pluripotency is achieved through insertion of transcription factor sequences through gene editing, concerns were raised over the possibility of mutation induction that could lead to further malignancies. This could potentially be averted using non-genomic vectors, but such approach would further lower the efficiency of IPSCs generation, potentially rendering it unviable [12]. There are also concerns regarding the plasticity of this type of cells. As potential high-rate proliferation and differentiation is often associated with malignant tumors, and some of the factors used to induce IPSC 


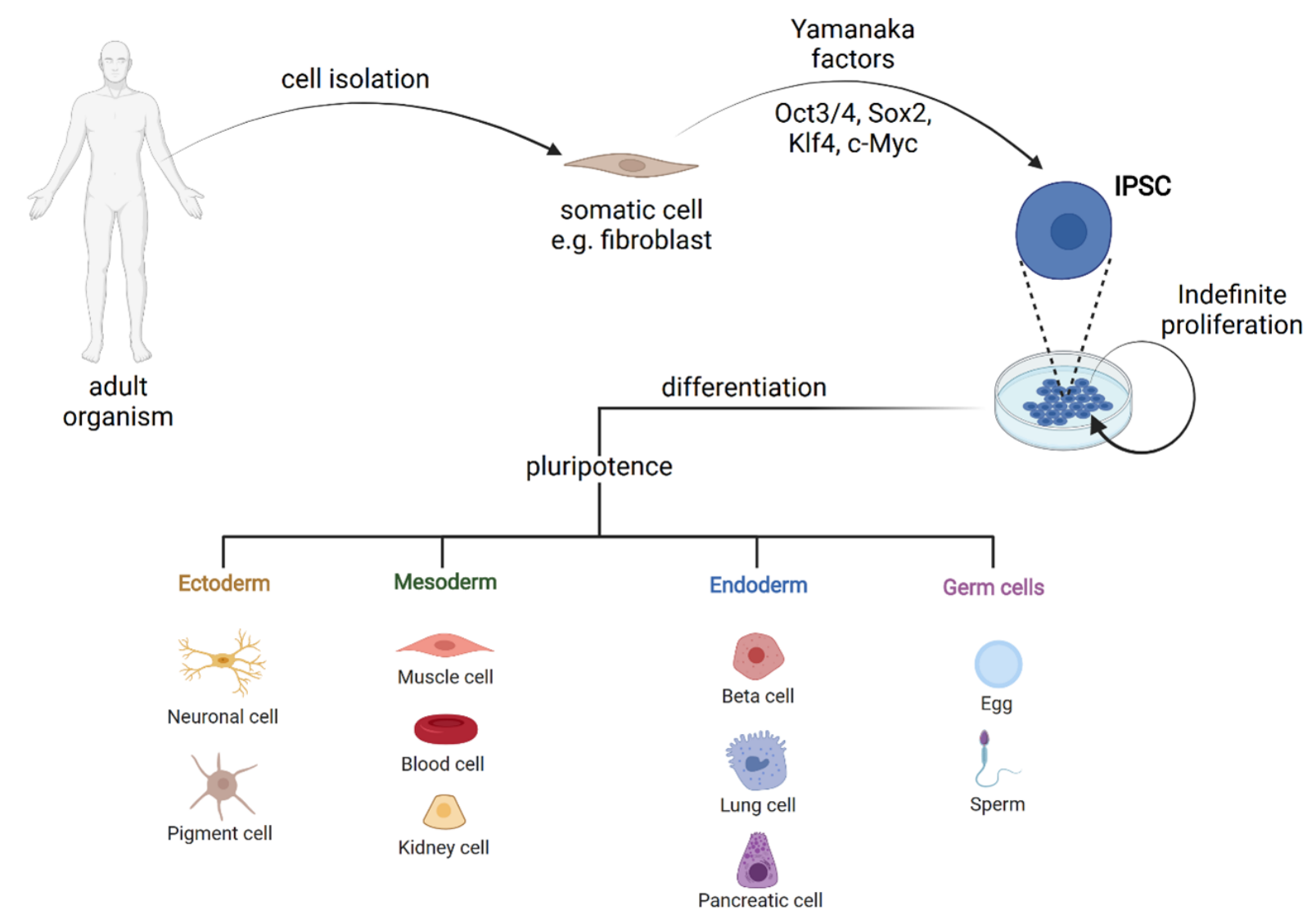

FIGURE 1 The process of IPSC creation and the extent of these cells' differentiation into a broad range of lineages. IPSCinduced pluripotent stem cells. Created with BioRender.com

pluripotency are known oncogenes, there is a possibility that administration of these cells to patients could result in cancerous progression [13]. Finally, the mechanisms governing stem cells plasticity and differentiation are still not fully elucidated. Hence, there are still no specific and precise methods that could confirm that reprogramming of the source somatic cells into IPSCs is complete and final. Application of such cells into a living host could result in unforeseen side effects due to the complexity and multifactorial nature of physiological cellular processes [14]. Summarizing, IPSCs have an enormous potential to be the solution for conditions requiring the use of pluripotent stem cells. However, a significant amount of basic and clinical studies still needs to be conducted to minimize the risk of adverse side effects of such therapies.

\section{Adult stem cells}

Adult stem cells are crucial to the functioning of the adult organism, facilitating cell turnover, as well as functioning and regeneration of a range of tissues. They are the only stem cell type readily available in humans in the post-birth period [15]. Hence, adult stem cells gain increasing attention of the scientific community and are indicated as an almost perfect source of cells for the fields of reconstruc- tive and regenerative medicine. While there are several types of those cells, the group that is most used and investigated in a clinical setting is known as mesenchymal stem cells (MSC). MSCs can be derived from multiple sources, with those sourced from the bone marrow already a basis of commonly used therapies for diseases such as leukemia or lymphoma [16]. However, obtaining of these cells is often associated with relatively invasive aspiration procedures, which prompts the search for alternative MSC sources. In the recent years, a significant attention is dedicated to adipose-derived stem cells (ASCs), which can be relatively easily isolated from fat tissue obtained through liposuction. While such approach is certainly less invasive than bone marrow aspiration, it needs to be noted that it is still based on a minor surgical procedure [17]. Further challenges related to the use of MSCs concern mostly their limited plasticity. Adult stem cells are multipotent, only being able to differentiate into a narrow range of lineages related to the tissue of origin [18]. Therefore, they can theoretically only be used to repair damage related to their niche. While some of them, including MSCs, can be forced to assume unrelated characteristic with the use of differentiation factors, there are other concerns associated with this approach. These mostly concern the in- 


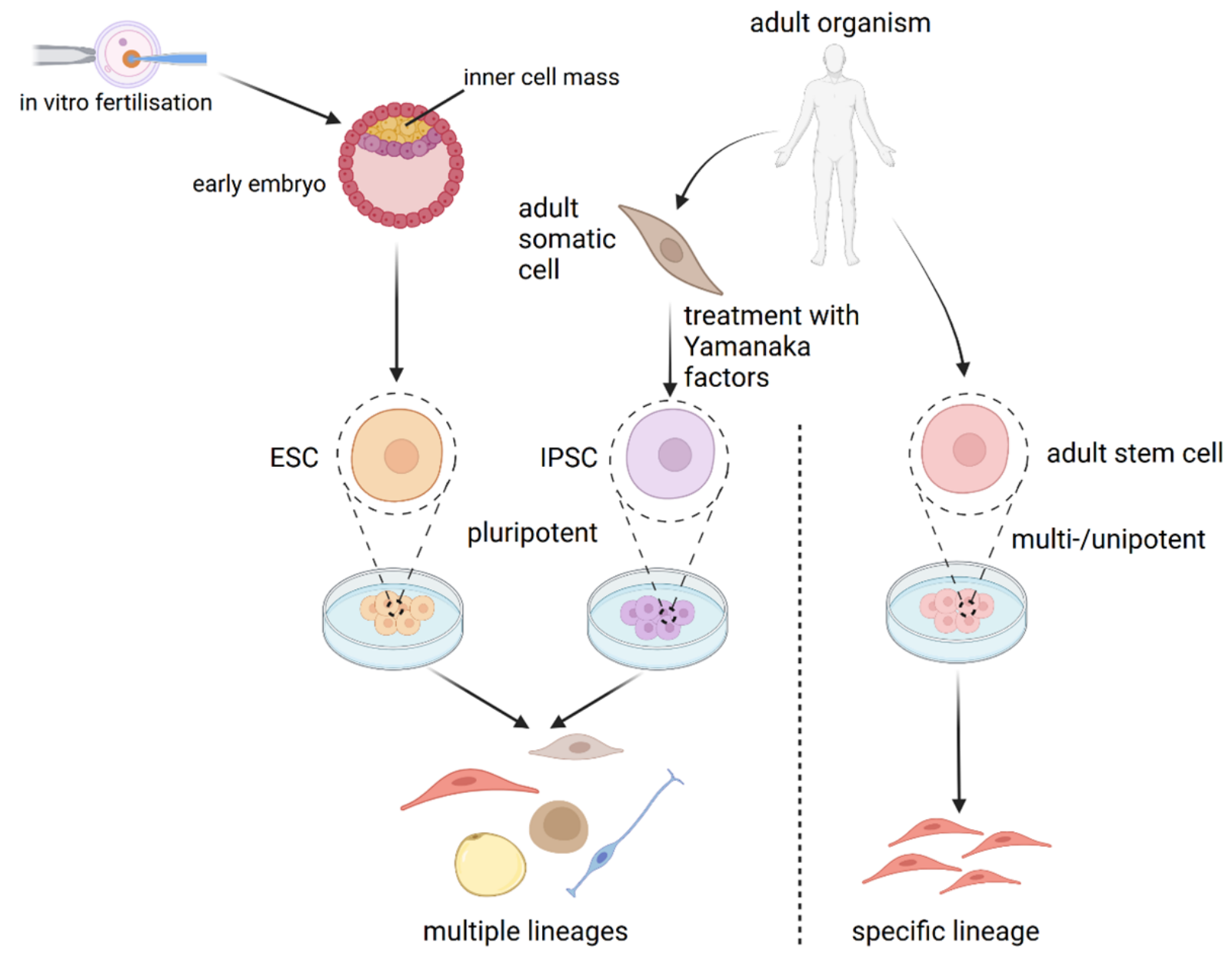

FIGURE 2 The summary graphical representation of the process of isolation and differentiation of the cell types described in this manuscript. ESC-embryonic stem cells; IPSC-induced pluripotent stem cell. Created with Biorender. Created with BioRender.com

complete knowledge of all the molecular mechanisms regulating the proliferation, functioning and differentiation of stem cells, which brings uncertainty about the extent of their dedication to the lineage assumed. A lack of full commitment to the target cellular identity could potentially cause lack of therapeutic effects, as well as even adverse side effects associated with the presence of abnormal cells in the location of the transplant [19]. Hence, while adult stem cells are certainly considered to be the future of modern medicine, there are several obstacles that need to be overcome before there wide scale introduction into the common clinical practice, mostly regarding the molecular mechanisms of the processes associated with their proliferation and differentiation. The cells described in this manuscript, as well as the processes of their isolation and differentiation, were presented on figure 2 .

\section{Conclusions}

There is no doubt that the discovery of stem cells is a major turning point in modern medicine, and they will continue to expand their foothold into new areas of clinical practice. However, there are several challenges that need to be overcome first, including the choice of the perfect source of stem cells, full elucidation of the mechanisms of stem cell differentiation and plasticity, and minimization of adverse side effect potential. Nonetheless, the focus of the scientific community on the topic of stem cells remains unhindered, bringing hope that all of the possible concerns will be addressed in the future.

\section{Ethical approval}

The conducted research is not related to either human or animal use.

\section{Acknowledgments}

Publication of this article was made possible by grant MH CZ - DRO (FNBr, 65269705), project AZV NV18-01-00544, AZV NV18-0800412 and KP-06-KOST/21-2019.

\section{Corresponding author}

Asss. prof. Ješeta Michal, Ph.D., Department of Obstetrics and Gynecology, University Hospital and Masaryk University, Obilni trh 11, 60200 Brno, Czech Republic, e-mail: jeseta@gmail.com.

\section{Conflict of interest}

The authors declare they have no conflict of interest. 


\section{References}

1. Charitos IA, Ballini A, Cantore S, Boccellino M, Di Domenico M, Borsani E, Nocini R, Di Cosola M, Santacroce L, Bottalico L. Stem Cells: A historical review about biological, religious, and ethical issues. Stem Cells Int. 2021;2021:9978837; DOI:10.1155/2021/9978837.

2. Nicolas P, Etoc F, Brivanlou AH. The ethics of human-embryoids model: a call for consistency. J Mol Med. 2021;99(4):569-79; DOI:10.1007/ S00109-021-02053-7.

3. Bongso A, Fong CY, Gauthaman K. Taking stem cells to the clinic: major challenges. J Cell Biochem. 2008;105(6):1352-60; DOI:10.1002/ JCB.21957.

4. Rippon HJ, Bishop AE. Embryonic stem cells. Cell Prolif. 2004;37(1):2334; DOI:10.1111/J.1365-2184.2004.00298.X.

5. Biswas A, Hutchins R. Embryonic Stem Cells. 2007;16(2):213-21; DOI:10.1089/SCD.2006.0081.

6. Martello G, Smith A. The nature of embryonic stem cells. 2014;30:64775; DOI:10.1146/ANNUREV-CELLBIO-100913-013116.

7. Lin G, Xie Y, OuYang Q, Qian X, Xie P, Zhou X, Xiong B, Tan Y, Li W, Deng L, Zhou J, Zhou D, Du L, Cheng D, Liao Y, Gu Y, Zhang S, Liu T, Sun Y, Lu G. HLA-matching potential of an established human embryonic Stem Cell Bank in China. Cell Stem Cell. 2009;5(5):461-5; DOI:10.1016/j. stem.2009.10.009.

8. Okita K, Yamanaka S. Induced pluripotent stem cells: opportunities and challenges. Philos Trans R Soc B Biol Sci. 2011;366(1575):2198-207; DOI:10.1098/RSTB.2011.0016.

9. Yamanaka S. Induced pluripotent stem cells: past, present, and future Cell Stem Cell. 2012;10(6):678-84; DOI:10.1016/J.STEM.2012.05.005.

10. Taylor CJ, Peacock S, Chaudhry AN, Bradley JA, Bolton EM. Generating an iPSC bank for HLA-matched tissue transplantation based on known donor and recipient HLA types. Cell Stem Cell. 2012;11(2):147-52; DOI:10.1016/J.STEM.2012.07.014.

11. Cao S, Loh K, Pei Y, Zhang W, Han J. Overcoming barriers to the clinical utilization of iPSCs: Reprogramming efficiency, safety and quality. Protein Cell. 2012;3(11):834-45; DOI:10.1007/s13238-012-2078-6.

12. Kang E, Wang X, Tippner-Hedges R, Ma H, Folmes CDL, Gutierrez NM, Lee Y, Van Dyken C, Ahmed R, Li Y, Koski A, Hayama T, Luo S, Harding CO, Amato P, Jensen J, Battaglia D, Lee D, Wu D, Terzic A, Wolf DP, Huang T, Mitalipov S. Age-Related Accumulation of somatic mitochondrial DNA mutations in adult-derived human iPSCs. Cell Stem Cell. 2016;18(5):625-36; DOI:10.1016/J.STEM.2016.02.005.

13. Riggs JW, Barrilleaux BL, Varlakhanova N, Bush KM, Chan V, Knoepfler PS. Induced pluripotency and oncogenic transformation are related processes. Stem Cells Dev. 2013;22(1):37-50; DOI:10.1089/ SCD.2012.0375

14. Zviran A, Hanna JH. Lucky iPSCs. Genome Biol. 2014;15(3):1-5; DOI:10.1186/GB4167

15. Young HE, Black AC. Adult stem cells 2004;276(1):75-102; DOI:10.1002/ ar.a.10134.

16. Stefanska K, Bryl R, Moncrieff L, Pinto N, Shibli JA, Dyszkiewicz-Konwińska M. Mesenchymal stem cells - a historical overview. Med J Cell Biol. 2020;8(2):83-7; DOI:10.2478/acb-2020-0010.

17. Yoshimura K, Shigeura T, Matsumoto D, Sato T, Takaki Y, Aiba-Kojima E, Sato K, Inoue K, Nagase T, Koshima I, Gonda K. Characterization of freshly isolated and cultured cells derived from the fatty and fluid portions of liposuction aspirates. J Cell Physiol. 2006;208(1):64-76; DOI:10.1002/ JCP.20636.

18. Wagers AJ, Weissman IL. Plasticity of adult stem cells. Cell. 2004;116(5):639-48; DOI:10.1016/S0092-8674(04)00208-9.

19. Kim E-H, Heo CY. Current applications of adipose-derived stem cells and their future perspectives. World J Stem Cells. 2014;6(1):65-8 DOI:10.4252/WJSC.V6.I1.65. 